Équations différentielles-Differential equations

\title{
Generalized Riesz basis property in the analysis of neutral type systems
}

\author{
Rabah Rabah ${ }^{1}$, Grigory Sklyar ${ }^{2}$ and Alexander Rezounenko ${ }^{3}$ \\ ${ }^{1}$ IRCCyN UMR 6597, 1 rue de la Noë, PB 92101, \\ F-44321 Nantes Cedex 3, France \\ Tél. : 02403769 15, Fax : 0240376930 \\ ${ }^{2}$ Institute of Mathematics, University of Szczecin, \\ 70-451 Szczecin, Wielkopolska 15, Poland \\ ${ }^{3}$ Department of Mechanics and Mathematics, Kharkov University, \\ 4 Svobody sqr., Kharkov, 61077, Ukraine \\ E-mails: rabah@irccyn.ec-nantes.fr, sklar@sus.univ.szczecin.pl, \\ rezounenko@univer.kharkov.ua
}

\begin{abstract}
The functional differential equation of neutral type is studied. We consider the corresponding operator model in Hilbert space $M_{2}=\mathbf{C}^{n} \times L_{2}\left(-1,0 ; \mathbf{C}^{n}\right)$ and prove that there exists a sequence of invariant finite-dimensional subspaces which constitute a Riesz basis in $M_{2}$. We also give an example emphasizing that the generalized eigenspaces do not form a Riesz basis.
\end{abstract}

\section{Bases généralisées de Riesz pour les systèmes de type neutre}

\section{Résumé}

On étudie une équation différentielle fonctionnelle de type neutre. Nous considérons le modèle opérationnel dans l'espace de Hilbert $M_{2}=\mathbf{C}^{n} \times L_{2}\left(-1,0 ; \mathbf{C}^{n}\right)$ et montrons qu'il existe dans cet espace une base de Riesz de sous-espaces de dimensions finies invariants par l'opérateur générateur infinitésimal du système. Nous donnons également un exemple précisant qu'il n'existe pas de base de Riesz de sous-espaces propres.

Key words. Neutral type systems, Riesz basis, Hilbert space, stability.

Mots clés. Systèmes de type neutre, base de Riesz, espace de Hilbert, stabilité.

2000 Mathematics Subject Classification. Primary 34K40; 47D06, 93C23.

\section{Version française abrégée}

Dans ce papier nous étudions l'équation différentielle fonctionnelle de type neutre (1), où $A_{-1}$ est une matrice constante telle que $\operatorname{det} A_{-1} \neq 0, A_{2}, A_{3}$ sont des matrices dont les éléments sont dans $L_{2}(-1,0)$. C'est une forme assez générale d'équation de type neutre qui apparaît, en particulier, quand on stabilise un système de type neutre, même si le système original est à retards ponctuels.

Le problème de la stabilité exponentielle de ce type de système est bien connu et étudié $[7,9]$. Afin d'analyser des propriétés plus fines de stabilité asymptotique de (1), 
la stabilité dite forte (cf. par exemple [3]), nous proposons de considérer (1) sous un forme opérationnelle abstraite et d'étudier ses propriétés spectrales dans des espaces de dimension infinie. Dans [7], l'approche est basée sur une description des systèmes de type neutre dans l'espace de fonctions $C\left([-1,0] ; \mathbf{C}^{n}\right)$. Une autre méthode [13] consiste à traiter le système dans l'espace $M_{2}=\mathbf{C}^{n} \times L_{2}\left(-1,0 ; \mathbf{C}^{n}\right)$, qui est un espace de Hilbert. On peut ainsi utiliser les techniques fines de la théorie des operateurs dans les espaces de Hilbert $[1,6]$. Dans ce contexte nous mettons en valeur l'importance du concept remarquable de base de Riesz dans l'analyse du modèle fonctionnel. Nous utilisons le modèle proposé dans [13] pour écrire le système (1) sous la forme (2), où l'operateur $\mathcal{A}$ est le générateur d'un $C_{0}$-semigroupe dans $M_{2}$. Nous montrons que $\mathcal{A}$ est un opérateur discret spectral [5] et nous étudions les conditions d'existence d'une base spectrale de Riesz.

Traditionnellement par base spectrale de Riesz on entend une base de vecteurs propres, de vecteurs propres généralisés ou de sous-espaces propres (cf. par exemple [4]). Il se trouve que l'operateur $\mathcal{A}$ ne possède pas une telle base dans le cas général (voir Exemple 8). Dans un premier temps nous étudions le cas de l'opérateur $\overline{\mathcal{A}}$ correspondant au système (1) avec $A_{2}(\theta)=A_{3}(\theta)=0$. On montre que les vecteurs propres et les vecteurs propres généralisés de cet opérateur forment une base de Riesz et donc une base de Riesz de sous-espaces propres généralisés. Ceci permet, par une technique d'approximation basée sur la théorie des perturbations des opérateurs linéaires [8], d'approximer une famille de sous-espaces invariants de $\mathcal{A}$ par ceux de $\overline{\mathcal{A}}$. Il vient alors le résultat suivant:

Théorème. L'opérateur $\mathcal{A}$ donné en (2), correspondant au système de type neutre (1), possède une suite de sous-espaces invariants, de dimensions finies, qui forment une base de Riesz de $\mathrm{M}_{2}$.

Ce résultat est d'une grande importance dans l'étude de la stabilité et de la stabilisabilité fortes des systèmes de type neutre.

\section{Introduction and operator model}

In this paper we analize the functional differential equation of neutral type

$$
\dot{x}(t)=A_{-1} \dot{x}(t-1)+\int_{-1}^{0} A_{2}(\theta) \dot{x}(t+\theta) d \theta+\int_{-1}^{0} A_{3}(\theta) x(t+\theta) d \theta
$$

where $A_{-1}$ is constant $n \times n$-matrix, $\operatorname{det} A_{-1} \neq 0, A_{2}, A_{3}$ are $n \times n$-matrix whose elements belong to $L^{2}(-1,0)$.

This equation occurs, for example, when a system of neutral type is stabilized. Even if the initial system contains pointwise delays only, then the set of natural feedback laws contains distributed delays (see e.g., $[11,10]$ ), so the corresponding closed-loop system takes the form (1).

The problem of exponential stability of systems like (1) is well studied [7,9]. In order to analize more subtle properties of stability (and stabilizability), namely strong asymptotic stability (see e.g. [3]), one needs to consider an operator model generated by the system (1) in some infinite dimensional space. Such an approach is also helpfull in studying of controllability and other problems of control theory.

In [7], the framework is based on the description of the neutral type system in the space of continuous functions $C\left([-1,0] ; \mathbf{C}^{n}\right)$. Another way $[13]$ is to treat the system in the space $M_{2}=\mathbf{C}^{n} \times L_{2}\left(-1,0 ; \mathbf{C}^{n}\right)$, which is a Hilbert space. That gives the possibility to use deep ideas and technique of the operator theory in Hilbert space $[1,6]$. 
In this context, we emphasize the importance of the remarkable concept of Riesz basis in the analysis of operator models. We use the model [13] to write the system (1) in the form

$$
\frac{d}{d t}\left(\begin{array}{c}
y(t) \\
z_{t}(\cdot)
\end{array}\right)=\mathcal{A}\left(\begin{array}{c}
y(t) \\
z_{t}(\cdot)
\end{array}\right), \quad \mathcal{A}\left(\begin{array}{c}
y \\
z(\cdot)
\end{array}\right)=\left(\begin{array}{c}
\int_{-1}^{0} A_{2}(\theta) \dot{z}(\theta) d \theta+\int_{-1}^{0} A_{3}(\theta) z(\theta) d \theta \\
d z(\theta) / d \theta
\end{array}\right),
$$

where the domain of $\mathcal{A}$ is given by $\mathcal{D}(\mathcal{A})=\left\{(y, z(\cdot)): z \in H^{1}\left(-1,0 ; C^{n}\right), y=z(0)-\right.$ $\left.A_{-1} z(-1)\right\} \subset M_{2}$ and the operator $\mathcal{A}$ is the generator of a $C_{0^{-}}$-semigroup. The relation between the solutions of the system (1) and the system (2) is, as usual, given by $z_{t}(\theta)=$ $x(t+\theta)$ for $\theta \in[-1,0]$. This operator model is an extension of the model introduced in $[13,14]$ in the case of pointwise delays. We show that $\mathcal{A}$ is a discrete spectral operator [5] and examine its spectral Riesz basis properties.

Traditionally, when studying the concept of spectral Riesz basis, one means the basis of eigen- and generalized eigenvectors, or generally, eigenspaces (see e.g., [4] and references therein). However, it turns out, that the operator $\mathcal{A}$ does not possess such a basis in general case (see Example 8). At the same time, we prove our main result:

Theorem The operator $\mathcal{A}$ (see (2)) corresponding to neutral type system (1) possesses the following generalized Riesz basis property:

There exists a sequence of invariant for $\mathcal{A}$ finite-dimensional subspaces which constitute a Riesz basis in $M_{2}$.

This result is crucial in the investigation of strong stability (stabilizability) of neutral type systems which is to be given in our forthcoming paper.

\section{Preliminary results}

In the sequel we will consider the matrix $A_{-1}$ in a Jordan basis and change the norm in $\mathbf{C}^{n}$ such that the corresponding eigen- and rootvectors of $A_{-1}$ form an orthogonal basis.

Let us denote by $\mu_{1}, \ldots, \mu_{\ell}, \mu_{i} \neq \mu_{j}$ if $i \neq j$, the eigenvalues of $A_{-1}$ and the dimensions of their rootspaces by $p_{1}, \ldots, p_{\ell}, \sum_{k=1}^{\ell} p_{k}=n$. Consider the points $\lambda_{m}^{(k)} \equiv$ $\ln \left|\mu_{m}\right|+i\left(\arg \mu_{m}+2 \pi k\right), m=1, . ., \ell ; k \in \mathbf{Z}$ and the circles $L_{m}^{k}$ of fixed radius $r \leq r_{0} \equiv$ $\frac{1}{3} \min \left\{\left|\lambda_{m}^{(k)}-\lambda_{i}^{(j)}\right|,(m, k) \neq(i, j)\right\}$ centered at $\lambda_{m}^{(k)}$.

Proposition 1. The spectrum of $\mathcal{A}$ consists of the eigenvalues only which are the roots of the equation $\operatorname{det} \Delta(\lambda)=0$, where

$$
\Delta_{\mathcal{A}}(\lambda)=\Delta(\lambda) \equiv-\lambda I+\lambda e^{-\lambda} A_{-1}+\lambda \int_{-1}^{0} e^{\lambda s} A_{2}(s) d s+\int_{-1}^{0} e^{\lambda s} A_{3}(s) d s .
$$

The corresponding eigenvectors of $\mathcal{A}$ are $\varphi=\left(\begin{array}{c}C-e^{-\lambda} A_{-1} C \\ e^{\lambda \theta} C\end{array}\right)$, with $C \in \operatorname{Ker} \Delta(\lambda)$.

There exists $N_{1}$ such that for any $k$, such that $|k| \geq N_{1}$, the total multiplicity of the roots of the equation $\operatorname{det} \Delta(\lambda)=0$, contained in the circle $L_{m}^{k}$, equals $p_{m}$.

Proposition 2. The resolvent of $\mathcal{A}$ is given by

$$
R(\mathcal{A}, \lambda)\left(\begin{array}{c}
x \\
\psi(\cdot)
\end{array}\right) \equiv\left(\begin{array}{c}
A_{-1} e^{-\lambda} \int_{-1}^{0} e^{-\lambda s} \psi(s) d s+\left(I-A_{-1} e^{-\lambda}\right) \Delta^{-1}(\lambda) D \\
\int_{0}^{\theta} e^{\lambda(\theta-s)} \psi(s) d s+e^{\lambda \theta} \Delta^{-1}(\lambda) D
\end{array}\right)
$$

where $D=D(x, \psi) \equiv x+\lambda e^{-\lambda} A_{-1} \int_{-1}^{0} e^{-\lambda s} \psi(s) d s-\int_{-1}^{0} A_{2}(s) \psi(s) d s-\int_{-1}^{0}\left\{\lambda A_{2}(\theta)+\right.$ $\left.A_{3}(\theta)\right\} e^{\lambda \theta}\left\{\int_{0}^{\theta} e^{-\lambda s} \psi(s) d s\right\} d \theta$. 
The proof of Proposition 2 is straightforward. Let us highlight the main steps of the proof of Proposition 1. It is easy to see from the explicit form of $R(\mathcal{A}, \lambda)$ and the compactness of embedding of $H^{1}\left(-1,0 ; \mathbf{C}^{n}\right)$ into $L_{2}\left(-1,0 ; \mathbf{C}^{n}\right)$, that $R(\mathcal{A}, \lambda)$ is compact. It gives that $\mathcal{A}$ has point spectrum only. Each eigenvalue is a root of $\operatorname{det} \Delta(\lambda)$ of finite multiplicity. Calculations give the form of eigenvectors of $\mathcal{A}$, taking into account the explicit definition of $D(\mathcal{A})$.

To describe the location of the spectrum of $\mathcal{A}$ we use Rouche theorem.

More precisely, for sufficiently large $k$ and any $m$ we show that $\left|f_{1}(\lambda)\right|>\left|f_{2}(\lambda)\right|$ for any $\lambda \in L_{m}^{k}$ and $f_{1}(\lambda) \equiv \operatorname{det}\left(A_{-1}-e^{-\lambda} I\right), f_{2}(\lambda) \equiv \operatorname{det}\left(A_{-1}-e^{-\lambda} I\right)-$ $\operatorname{det}\left(A_{-1}-e^{-\lambda} I+e^{\lambda} \int_{-1}^{0} e^{\lambda s} A_{2}(s) d s+e^{\lambda} \lambda^{-1} \int_{-1}^{0} e^{\lambda s} A_{3}(s) d s\right)$. Thus, $f_{1}-f_{2}$ has the same number of roots inside $L_{m}^{k}$ as function $f_{1}$.

Proposition 3. The operator $\mathcal{A}$ generates a $C_{0}$-semigroup in $M_{2}$.

In the particular case when $A_{2}(\theta)=A_{3}(\theta) \equiv 0$, we will use the notation $\overline{\mathcal{A}}$ for $\mathcal{A}$. We will show that the properties of $\overline{\mathcal{A}}$ can be expressed in terms of the properties of matrix $A_{-1}$ only. The basis properties of the operator $\mathcal{A}$ will be deduced from the ones of $\overline{\mathcal{A}}$.

\section{Basis property of eigen- and rootvectors of the operator $\overline{\mathcal{A}}$.}

Let $\nu_{m}$ be the number of Jordan blocks, corresponding to $\mu_{m} \in \operatorname{spec}\left(A_{-1}\right)$. Denote by $p_{m, j}, j=1, . ., \nu_{m}, \sum_{j=1}^{\nu_{m}} p_{m, j}=p_{m}$, the orders of these blocks and by $\left\{C_{m, j}^{0}, \ldots, C_{m, j}^{p_{m, j}-1}\right\}$ the orthonormal system of corresponding eigen- and rootvectors i.e., $A_{-1} C_{m, j}^{0}=\mu_{m} C_{m, j}^{0}$, $A_{-1} C_{m, j}^{d}=\mu_{m} C_{m, j}^{d}+C_{m, j}^{d-1}, d=1, . ., p_{m, j}-1 ; j=1, . ., \nu_{m}$. If there exists $k \in\{1, \ldots, \ell\}$ such that $\mu_{k}=1 \in \operatorname{spec}\left(A_{-1}\right)$ we denote by $K$ the rootspace of $A_{-1}$ corresponding to the eigenvalue 1 and put $K=\{0\}$ otherwise. Finally, let $K_{1}=K^{\perp}=\operatorname{Lin}\left\{C_{m, j}^{d}, m \in\right.$ $\left.\{0, \ldots, \ell\}: \mu_{m} \neq 1 ; j=1, \ldots, \nu_{m} ; d=0, \ldots, p_{m, j}-1\right\}$. In order to describe eigen- and rootvectors of the operator $\overline{\mathcal{A}}$ (see Theorem 5 ) we need the following lemma.

Lemma 4. i) Let us put $\nu(1)=0$ if $1 \notin \operatorname{spec}\left(A_{-1}\right)$ and $\nu(1)=\nu_{k}$ if for some $k \in\{0, \ldots, \ell\}: \mu_{k}=1$. For any $y \in \mathbf{C}^{n}$ there exists an unique polynomial vector $P_{y}(\theta)$ of the form

$$
P_{y}(\theta)=\sum_{j=1}^{\nu(1)} \sum_{d=0}^{p_{k, j}-1} C_{k, j}^{d}\left(\alpha_{y}^{0, j} \frac{\theta^{p_{k, j}-d}}{\left(p_{k, j}-d\right) !}+\alpha_{y}^{1, j} \frac{\theta^{p_{k, j}-d-1}}{\left(p_{k, j}-d-1\right) !}+\cdots+\alpha_{y}^{p_{k, j}-d-1, j} \theta\right)+\gamma_{y},
$$

such that $\gamma_{y} \in K_{1}$ and $y=P_{y}(0)-A_{-1} P_{y}(-1)$.

ii) The mapping $y \stackrel{D}{\rightarrow} P_{y}(\cdot)$ is a linear operator $D: \mathbf{C}^{n} \rightarrow H^{1}\left(-1,0 ; \mathbf{C}^{n}\right)$.

Remark. In particular case when $1 \notin \operatorname{spec}\left(A_{-1}\right)$ Lemma 4 gives $P_{y}(\theta)=\left(I-A_{-1}\right)^{-1} y$.

Theorem 5. The spectrum of $\overline{\mathcal{A}}$ consists of points $\lambda_{m}^{(k)}$ with corresponding eigen-and rootvectors

$$
\left(\begin{array}{c}
0 \\
e^{\lambda_{m}^{(k)} \theta} C_{m, j}^{0}
\end{array}\right),\left(\begin{array}{c}
0 \\
e^{\lambda_{m}^{(k)} \theta}\left(C_{m, j}^{0} \theta+C_{m, j}^{1}\right)
\end{array}\right), \ldots,\left(\begin{array}{c}
0 \\
e^{\lambda_{m}^{(k)} \theta}\left(C_{m, j}^{0} \frac{\theta^{p_{m, j}-1}}{\left(p_{m, j}-1\right) !}+\ldots+C_{m, j}^{p_{m, j}-1}\right)
\end{array}\right),
$$


$m=1, \ldots, \ell, k \in \mathbf{Z}$, and also of the point 0 for which one can choose eigen- or rootvectors of the form

$$
\left(\begin{array}{c}
e_{i} \\
P_{e_{i}}(\theta)
\end{array}\right)
$$

where $\left\{e_{i}\right\}_{i=1}^{n}$ is an arbitrary orthogonal basis in $\mathbf{C}^{n}$ and the polynomial $P_{y}(\theta)$ is described in Lemma 4 .

The collection (5) and (6) constitutes a Riesz basis in $M_{2}$ which becomes an orthogonal basis if we choose the equivalent norm

$$
\|\left(y, z(\cdot)\left\|_{1}^{2}=\right\| y\left\|^{2}+\int_{-1}^{0}\right\| T\left(P_{y}(\theta)-z(\theta)\right) \|^{2} d \theta\right.
$$

where $T$ is a bounded operator in $L_{2}\left(-1,0 ; \mathbf{C}^{n}\right)$ with bounded inverse.

\section{Basis property of finite-dimensional invariant subspaces}

Theorem 6. There exists $N_{0}$ large enough, such that for any $N \geq N_{0}$,

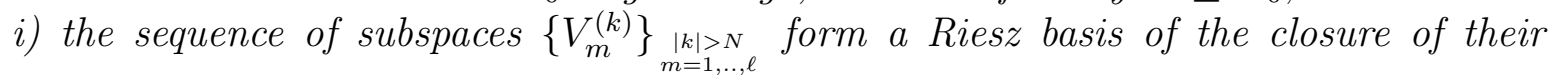
linear span, say $\mathcal{L}_{N}$. Here $V_{m}^{(k)} \equiv P_{m}^{(k)} M_{2}$ and $P_{m}^{(k)} M_{2}=\frac{1}{2 \pi i} \int_{L_{m}^{(k)}} R(\mathcal{A}, \lambda) d \lambda$ are spectral projectors; $L_{m}^{(k)}$ are circles defined in Section 2;

ii) $\operatorname{codim} \mathcal{L}_{N}=(2 N+1) n+n=2(N+1) n$.

To prove item i) we estimate the norm of the difference $P_{m}^{(k)}-\bar{P}_{m}^{(k)}$ using the explicit form of resolvent (see (4)). Then we choose $N_{0}$ big enough such that $\sum_{m=1}^{\ell} \sum_{|k|>N_{0}} \| P_{m}^{(k)}-$ $\bar{P}_{m}^{(k)} \|^{2}<1$. The last estimate means that the sequences of subspaces $\left\{V_{m}^{(k)}\right\}_{\substack{|k|>N \\ m=1, . .,}}$ and $\left\{\bar{V}_{m}^{(k)}\right\}_{\substack{|k|>N \\ m=1, ., \ell}}$ are quadratically close. Theorem 5.2 [6] (see also Th.2.20 and Corollary 2.22 in [8]) and the basis property of $\left\{\bar{V}_{m}^{(k)}\right\}_{\substack{k \in \mathbf{Z} \\ m=1, .,}}$ (see Theorem 5) prove item i).

To prove item ii) we use that the sequence $\left\{\bar{V}_{m}^{(k)}\right\}_{\substack{k \in \mathbf{Z} \\ m=1, \ldots, \ell}}$ form an orthogonal basis in $M_{2}$ (see Theorem 5) and $\left\{\bar{V}_{m}^{(k)}\right\}_{\substack{|k| \leq N \\ m=1, \ldots, \ell}} \cup\left\{V_{m}^{(k)}\right\}_{\substack{|k|>N \\ m=1, \ldots,}}$ is quadratically close to the sequence $\left\{\bar{V}_{m}^{(k)}\right\}_{\substack{k \in \mathbf{Z} \\ m=1 . ., \ell}}$ (see item i). Hence $\left\{\bar{V}_{m}^{(k)}\right\}_{\substack{|k| \leq N \\ m=1, . .,}} \cup\left\{V_{m}^{(k)}\right\}_{\substack{|k|>N \\ m=1, . ., \ell}}^{\substack{m=1, ., \ell \\(k)}}$ form a Riesz basis in $M_{2}$ and $\operatorname{dim}\left\{\bar{V}_{m}^{(k)}\right\}_{\substack{|k| \leq N \\ m=1, . ., \ell}}=2(N+1) n$.

The proof of Theorem 6 is complete.

The main result of our work is the following

Theorem 7. There exists a sequence of invariant for $\mathcal{A}$ finite-dimensional subspaces which constitute a Riesz basis in $M_{2}$.

More precisely, these subspaces are $\left\{V_{m}^{(k)}\right\}_{\substack{|k|>N \\ m=1, \ldots, \ell}}$ (see Theorem 6) and a $2(N+1) n$ dimensional subspace spaned by all eigen- and rootvectors, corresponding to all eigenvalues of $\mathcal{A}$, which are outside of all circles $L_{m}^{(k)},|k|>N, m=1, . ., \ell$.

\section{Proof of Theorem 7.}

The proof consists of three steps.

Step 1. Let us set $X_{1}=\mathcal{L}_{N}$, where $\mathcal{L}_{N}$ is defined in Theorem 6. The subspace $X_{1}$ is of finite co-dimension and invariant for $\mathcal{A}$. 
One can show that $M_{2}$ can be split into the direct sum $M_{2}=X_{1}+X_{2}$, and the operator $\mathcal{A}$ can be presented in the triangular form $\mathcal{A}=\left(\begin{array}{cc}A_{11} & A_{12} \\ 0 & A_{22}\end{array}\right)$, where $A_{11} \equiv P_{1} \mathcal{A} P_{1}: X_{1} \rightarrow X_{1}$, $A_{22} \equiv P_{2} \mathcal{A} P_{2}: X_{2} \rightarrow X_{2}, A_{12} \equiv P_{1} \mathcal{A} P_{2}: X_{2} \rightarrow X_{1}$. Here $P_{i}$ are projectors on $X_{i}$ along $X_{j}, j \neq i$.

Step 2. We prove that $\operatorname{spec}\left(A_{11}\right) \cap \operatorname{spec}\left(A_{22}\right)=\emptyset$.

Step 3. Let us show that $M_{2}$ can be split into the direct sum $M_{2}=X_{1}+\hat{X}_{2}$, with an invariant $\hat{X}_{2}$. Consider finite-dimensional operator $A_{22}$. There exists $\operatorname{spec}\left(A_{22}\right)=\left\{\hat{\mu}_{1}, . ., \hat{\mu}_{s}\right\}$ with the total multiplicity equals to $\operatorname{dim} X_{2}=\operatorname{codim} X_{1}$.

Consider $h$ - an eigenvector of $A_{22}$ corresponding to $\hat{\mu}$ and find $y \in X_{1}$ such that $\mathcal{A}\left(\begin{array}{l}y \\ h\end{array}\right)=$ $\hat{\mu}\left(\begin{array}{l}y \\ h\end{array}\right)$. Such $y$ is given by $y=-\left(A_{11}-\hat{\mu} I\right)^{-1} A_{12} h$ (see the triangular form of $\mathcal{A}$ ). This is due to the property $\operatorname{spec}\left(A_{11}\right) \cap \operatorname{spec}\left(A_{22}\right)=\emptyset$, which implies $\hat{\mu} \notin \operatorname{spec}\left(A_{11}\right)$. Exactly in the same way one can find all rootvectors of $\mathcal{A}$ for all $\hat{\mu}$. Hence the number of eigenand rootvectors of $\mathcal{A}$ corresponding to $\operatorname{spec}\left(A_{22}\right)$ is equal to $\operatorname{dim} X_{2}=2(N+1) n$. By the construction, the linear span of these vectors gives an invariant $\hat{X}_{2}$. Now Theorem 6 completes the proof of Theorem 7 . We emphasize that the operator $\mathcal{A}$ does not possess in general case a Riesz basis of generalized eigenspaces. We illustrate this on the following

Example 8. Consider the particular case of the system (1):

$$
\dot{x}(t)=A_{-1} \dot{x}(t-1)+A_{0} x(t), \quad A_{-1}=\left(\begin{array}{ll}
1 & 1 \\
0 & 1
\end{array}\right), \quad A_{0}=\left(\begin{array}{ll}
\alpha & 0 \\
0 & \beta
\end{array}\right) .
$$

One can check that the characteristic equation is $\operatorname{det} \Delta(\lambda)=\left(\alpha-\lambda+\lambda e^{-\lambda}\right)\left(\beta-\lambda+\lambda e^{-\lambda}\right)=0$ and for $\alpha \neq \beta$ there are two sequences of eigenvectors, such that $\left\|v_{n}^{1}-v_{n}^{2}\right\| \rightarrow 0$, as $n \rightarrow \infty$. By definition, such vectors can not form a Riesz basis. More precisely, assume opposite i.e., that $\left\{v_{n}^{i}\right\}$ form a Riesz basis. Then there exists [6] an orthonormal basis $\left\{\eta_{n}^{i}\right\}$ and a bounded operator $T$ such that $T v_{n}^{i}=\eta_{n}^{i}, i=1,2 ; n \in \mathbf{Z}$. We have that $\left\langle T\left(v_{n}^{1}-v_{n}^{2}\right), \eta_{n}^{1}\right\rangle=\left\langle\eta_{n}^{1}-\eta_{n}^{2}, \eta_{n}^{1}\right\rangle=1$ for all $n$. On the other hand, one gets $\left\langle T\left(v_{n}^{1}-v_{n}^{2}\right), \eta_{n}^{1}\right\rangle \leq$ $\|T\| \cdot\left\|v_{n}^{1}-v_{n}^{2}\right\| \cdot\left\|\eta_{n}^{1}\right\| \rightarrow 0$, as $n \rightarrow \infty$. We arrive to a contradiction.

Acknowledgements. This work was realized with the financial support of Region Pays de la Loire, Ecole Centrale de Nantes (France) and grant 5 PO3A 03021 (KBN, Poland).

\section{REFERENCES}

1. Akhiezer N.I. and Glazman I.M. Theory of linear operators in Hilbert space. Transl. from the Russian. Repr. of the 1961 and 1963 transl. (English) New York, NY: Dover Publications. 218 p.

2. Bellman R. and Cooke K.L. Differential-difference equations, in "Mathematics in Science and Engineering". Vol. 6. New York-London: Academic Press, XVI, 1963.

3. Brumley W.E., On the asymptotic behavior of solutions of differential-difference equations of neutral type, J. Differential Equations 7(1970), 175-188.

4. Curtain R.F. and Zwart H., An introduction to infinite-dimensional linear systems theory, in "Texts in Applied Mathematics", Vol. 21, Springer-Verlag, New York, 1995. 
5. Dunford N. and Schwartz J.T., "Linear operators, Part II: Spectral theory. Self adjoint operators in Hilbert space", Interscience, 1963.

6. Gohberg I.C. and Krein M.G. Introduction to the theory of linear nonselfadjoint operators (English) Translations of Mathematical Monographs, 18, Providence, RI: AMS. XV, 378 p. (1969).

7. Hale J. and Verduyn Lunel S. M, "Theory of functional differential equations", SpringerVerlag, New York, 1993.

8. Kato T., Perturbation theory for linear operators, Springer Verlag, 1980.

9. Kolmanovskii V. and Myshkis A., Introduction to the theory and applications of functional differential equations, Mathematics and its Applications, 463, Dordrecht, Kluwer Academic Publishers. 1999.

10. O'Connor D. A. and Tarn T. J., On stabilization by state feedback for neutral differential equations, IEEE Transactions on Automatic Control, Vol. AC-28(1983), no 5, pp. 615-618.

11. Pandolfi L., Stabilization of neutral functional differential equations, J. Optimization Theory and Appl. 20(1976), no. 2, 191-204.

12. Rabah R. and Sklyar G. M., On a class of strongly stabilizable systems of neutral type, (submitted).

13. Yamamoto Y. and Ueshima S., A new model for neutral delay-differential systems, Internat. J. Control 43(1986), no. 2, 465-471.

14. Yamamoto Y., Reachability of a class of infinite-dimensional linear systems: an external approach with applications to general neutral systems, SIAM J. Control Optim. 27(1989), no. 1, pp. 217-234. 